\title{
Huge and recurrent undifferentiated carcinoma with osteoclast- like giant cells of the pancreas
}

\author{
Lan Zhang', Jeong Min Lee ${ }^{2}$, Jeong Hee Yoon ${ }^{2}$, Ijin Joo², Hyo Jin Kang ${ }^{2}$, Joon Koo Han ${ }^{2}$, Sun Kyung \\ Jeon $^{2}$
}

${ }^{1}$ Department of MRI, The First Affiliated Hospital of Henan University of Chinese Medicine, Zhengzhou 450000, China; ${ }^{2}$ Department of Radiology, Seoul National University Hospital, 103 Daehak-ro, Jongno-gu, Seoul 110-744, South Korea

Correspondence to: Jeong Min Lee, MD. Department of Radiology, Seoul National University Hospital, 103 Daehak-ro, Jongno-gu, Seoul 110-744, South Korea. Email: jmlshy2000@gmail.com.

Submitted Mar 22, 2018. Accepted for publication May 08, 2018.

doi: 10.21037/qims.2018.05.05

View this article at: http://dx.doi.org/10.21037/qims.2018.05.05

\section{Introduction}

Undifferentiated carcinoma with osteoclast-like giant cells (OGCs) of the pancreas (UCOGCP) is a rare pancreatic tumor originated from pancreatic ductal epithelium. It is considered as a variant subtype of pancreatic ductal adenocarcinoma (PDAC), accounting for less than $1 \%$ of pancreatic malignant neoplasms $(1,2)$. Most UCOGCPs are found to have a large tumor upon detection, which are frequently unresectable. Even treated with surgical removal, UCOGCP has a tendency of early recurrence. Hereby we report a case of a large UCOGCP with recurrence shortly after surgical excision.

\section{Case presentation}

A 57-year-old male was admitted with epigastric pain and weight loss for 1 month. Lab examinations showed carbohydrate antigen (CA)-199 of $3 \mathrm{U} / \mathrm{mL}$, carcinoembryonic antigen (CEA) of $2.4 \mathrm{ng} / \mathrm{mL}$, and alpha-fetoprotein (AFP) of $0.8 \mu \mathrm{g} / \mathrm{L}$. Most indices of laboratory tests were normal except for slight anemia. Abdominal computed tomography (CT) revealed an approximately $12 \mathrm{~cm} \times 9 \mathrm{~cm} \times 8 \mathrm{~cm}$, roundlike, well-defined, cystic and solid mass in the left upper abdomen (Figure 1A). Contrast-enhanced CT demonstrated multiple cystic vesicles with septations and inhomogeneous enhancement of the solid portion within the tumor (Figure $1 B$ ). Magnetic resonance imaging (MRI) demonstrated a large miscellaneous signal intensity mass, presenting with slightly elevated signal intensity on diffusion-weighted imaging (DWI) (Figure 1C,D,E). After administration of gadolinium-diethylenetriaminepentaacetic acid (Gd-DTPA), the septations and capsule of the tumor enhanced markedly (Figure $1 F, G$ ). The tumor appeared to have originated from the pancreatic tail with invasion to the spleen, abutting parts of the stomach and colon. ${ }^{18} \mathrm{~F}$-fluorodeoxyglucose $\left({ }^{18} \mathrm{~F}\right.$-FDG) PET-CT revealed high uptake within the tumor (Figure 1H). No obvious lymphadenopathy, ascites, or distant metastases was detected on PET-CT.

The patient underwent pancreatectomy with splenectomy and partial gastrectomy, as well as resection of the partial colon. During operation, a $12 \mathrm{~cm} \times 9 \mathrm{~cm} \times 9 \mathrm{~cm}$ encapsulated multilocular cystic and solid mass was located in the tail of pancreas with adhesion to the surrounding tissue. There were cysts filled with hemorrhagic and necrotic contents on the cut surface (Figure 2A). On histological microscope, the tumor was found pleomorphic mononuclear cells with distributed multinucleated OGC (Figure 2B). The pleomorphic mononuclear cells exhibited bizarre features with different irregular shaped nuclei, prominent nucleoli with mitotic figures, and abundant cytoplasm. On the other hand, the OGC hardly had any phagocytosis and lacked atypical features. Immunohistology revealed that OGCs were positive for $\mathrm{CD} 68$, which is a characteristic histiocytic marker of OGC. Therefore, the diagnosis of UCOGCP was able to be established.

However, follow-up CT discovered metastases in both lungs 3 months after the operation. Contrast-enhanced 

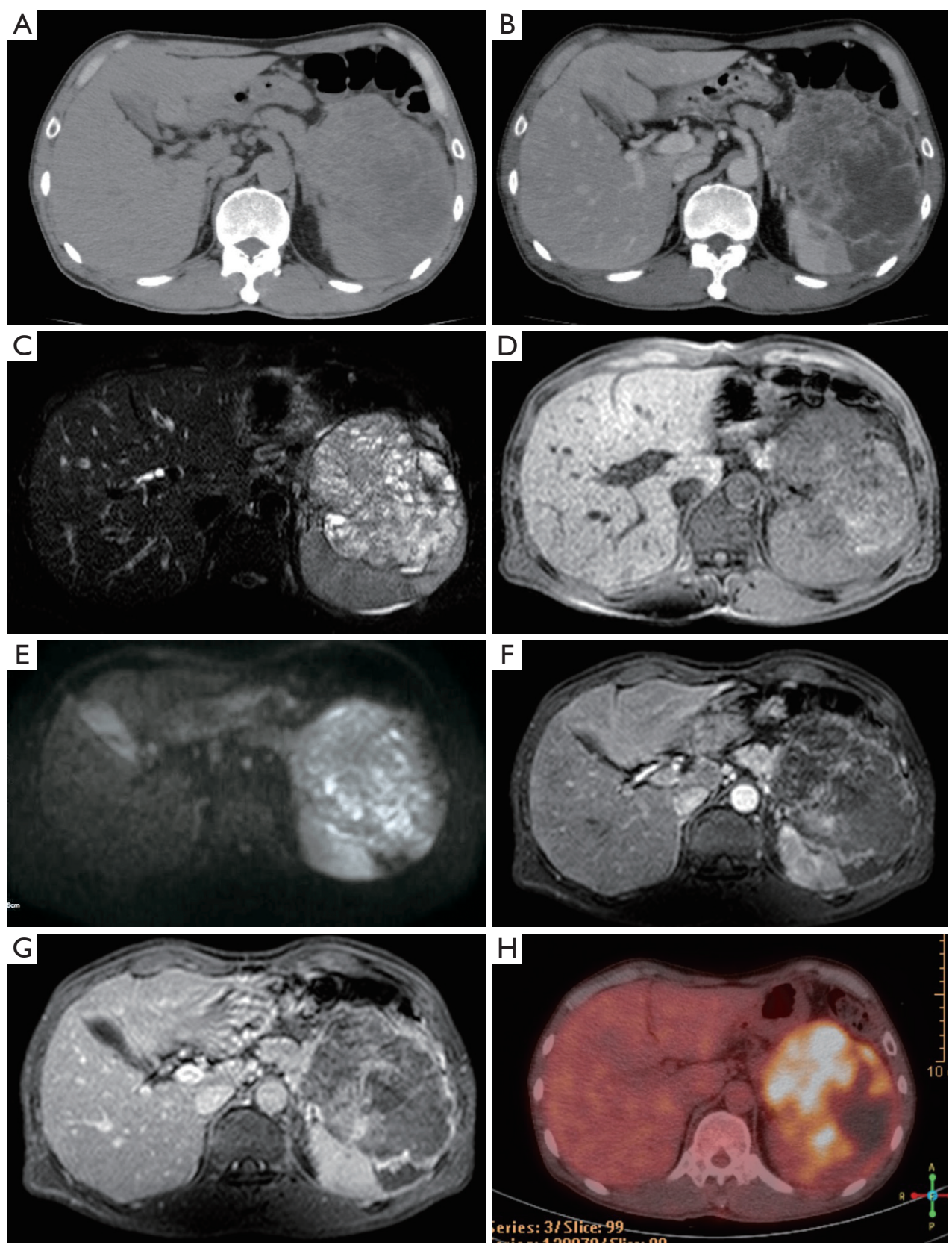

Figure 1 Huge undifferentiated carcinoma with osteoclast-like giant cells prior to operation. (A) CT plain scan shows a huge, well-defined, cystic and solid mass in the left upper quadrant of the abdomen; (B) contrast-enhanced CT demonstrates multiple cystic vesicles with septations with inhomogeneous enhancement of the solid portion within the tumor; (C) T2-weighted imaging (T2WI) shows a large mass with miscellaneous high signal intensity; (D) T1-weighted imaging (T2WI) shows patchy high signal within the low signal intensity tumor; (E) diffusion-weighted imaging (DWI) shows a mass with slightly high signal intensity; (F,G) contrast-enhanced MRI demonstrates that the septations and capsule of the tumor enhanced markedly; $(\mathrm{H}){ }^{18}$ F-FDG PET-CT shows the high uptake within the tumor.

abdominal CT revealed a $12 \mathrm{~cm} \times 10 \mathrm{~cm} \times 7.5 \mathrm{~cm}$, huge, multifocal, recurrent mass with inhomogeneous enhancement in the left abdomen, surrounding the left kidney with invasion to the partial kidney (Figure $3 A, B$ ). The patient continued to be treated with chemotherapy and was under the close surveillance. 

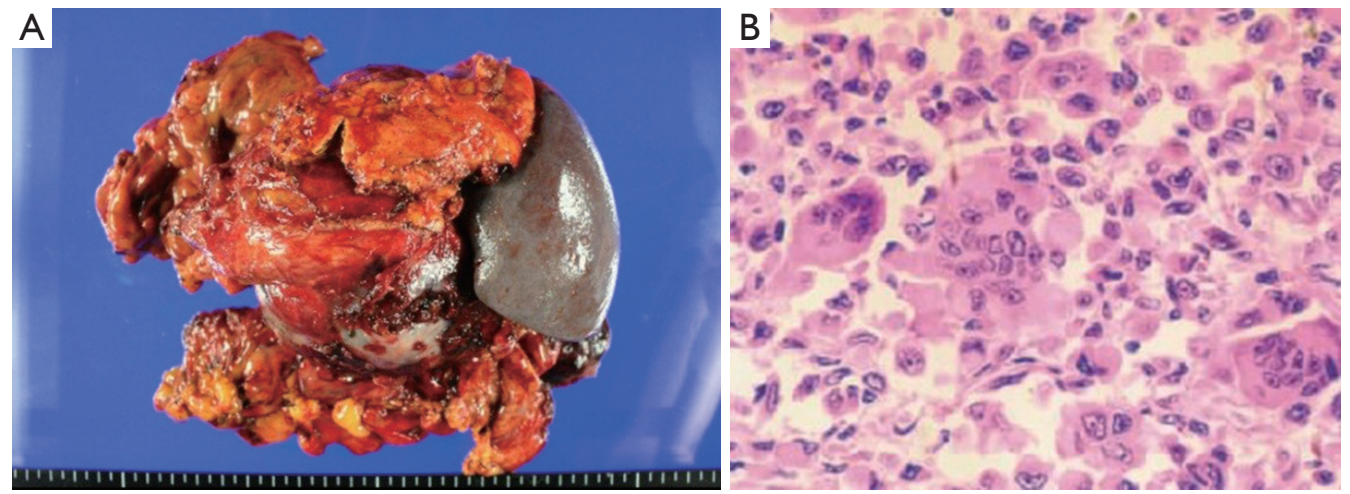

Figure 2 Gross findings show a $12 \mathrm{~cm} \times 9 \mathrm{~cm} \times 9 \mathrm{~cm}$ mass in the pancreatic tail along with cysts filled with hemorrhage and necrosis on the cut surface (A), pathologic examination reveals that the tumor is composed of pleomorphic mononuclear cells and osteoclast-like giant cells (hematoxylin and eosin staining, magnification $\times 20)(B)$.
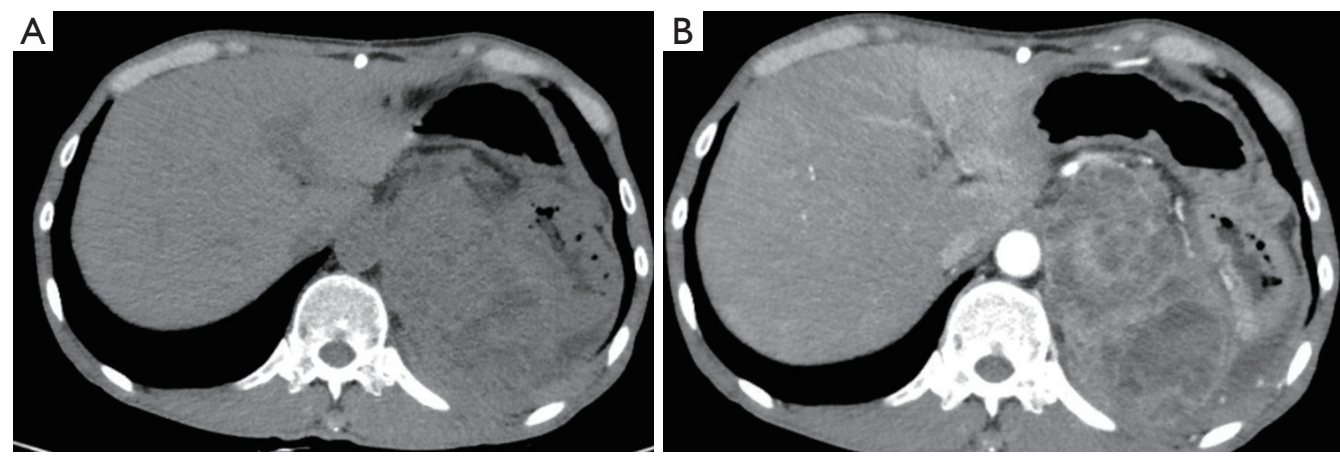

Figure 3 Recurrent undifferentiated carcinoma with osteoclast-like giant cells. (A) CT plain scan depicts a huge, multifocal, recurrent intraperitoneal mass in the left abdomen; (B) contrast-enhanced CT shows a large cystic and solid mass with inhomogeneous enhancement.

\section{Discussion}

Although UCOGCP is considered as a variance of PDAC, their features have been shown to be different from PDAC. The clinical features are $(3,4)$ : (I) non-specific symptoms and normal laboratory tests for CEA and CA199; (II) predilection toward males with an average age of 60 years; (III) arise from any portion of the pancreas, and more frequently from the body and tail of the pancreas; (IV) usually large with an average diameter of $12 \mathrm{~cm}$; (V) solid mass or solid and cystic mass presenting with hemorrhage and necrosis; (VI) more invasive than PDACs. In our case, the patient was a 57 -year-old male with non-specific clinical symptoms. The tumor originated from the tail of the pancreas, and the original mass prior to the operation and the recurrent mass were both over $10 \mathrm{~cm}$.

Since the growth of tumor is very rapid in a short period of time, UCOGCP often appears as a heterogeneous mass with distinct hemorrhage and necrosis. An inhomogeneous appearance with cystic structures and nonuniform enhancement is the typical radiological finding of UCOGCP $(5,6)$. MRI demonstrated a well-demarcated, heterogeneous cystic tumor with prominent enhancement of the septations and the capsule in our case. The previous studies also reported that lymph nodes involvement is less common in UCOGCPs $(7,8)$. Indeed, our case also showed no obvious lymph node involvement. The sensitivities of CT, MRI, and endoscopic ultrasonography for the detection of UCOGCP have been shown to be similar to that of PDAC (9). However, the diagnosis of UCOGCP cannot be made by $\mathrm{CT}$ or MRI alone, requiring confirmation through histopathology and immunohistochemistry.

UCOGCP is usually composed of pleomorphic mononuclear cells and non-neoplastic OGCs. The presence of non-neoplastic OGC is the histological distinguishing 
feature of UCOGCP. OGC is found to lack morphological atypia and proliferative activity. In addition, the presence of OGCs may predict a more favorable prognosis than undifferentiated pancreatic carcinomas without OGC (10).

The differential diagnoses of UCOGCP include solid pancreatic tumors, such as PDACs and pancreatic neuroendocrine tumors, and cystic pancreatic neoplasms, such as serous and pancreatic mucinous cystadenocarcinomas or pseudocysts. PDAC appears to be uniform density or intensity with gradual enhancement on CT and MRI, frequently with dilation of the common bile duct and pancreatic duct with rare occurrences of hemorrhage and necrosis (11). Mucinous cystadenocarcinoma of the pancreas usually occurs in old females and originates from the tail of the pancreas with solitary or multiple cysts.

\section{Acknowledgements}

None.

\section{Footnote}

Conflicts of Interest: The authors have no conflicts of interest to declare.

Informed Consent: Written informed consent was obtained from the patient for publication of this manuscript and any accompanying images.

\section{References}

1. Bosman FT, Carneiro F, Hruban RH, Theise ND. WHO Classification of Tumors of the Digestive System. 4th edition. Lyon: International Agency for Research on Cancer (IARC), 2010:281-95.

2. Maksymov V, Khalifa MA, Bussey A, Carter B, Hogan M. Undifferentiated (anaplastic) carcinoma of the pancreas with osteoclast-like giant cells showing various degree of pancreas duct involvement. A case report and literature

Cite this article as: Zhang L, Lee JM, Yoon JH, Joo I, Kang HJ, Han JK, Jeon SK. Huge and recurrent undifferentiated carcinoma with osteoclast-like giant cells of the pancreas. Quant Imaging Med Surg 2018;8(4):457-460. doi: 10.21037/ qims.2018.05.05 review. JOP 2011;12:170-6.

3. Gao HQ, Yang YM, Zhuang Y, Liu P. Locally advanced undifferentiated carcinoma with osteoclast-like giant cells of the pancreas. World J Gastroenterol 2015;21:694-8.

4. Verbeke CS, Menon KV. Osteoclast-like giant cell tumour of the pancreas: an undifferentiated carcinoma of duct epithelial origin. Pancreatology 2006;6:254; author reply 254.

5. Njoumi N, Elalami FH, Attolou G, Saoud O, Elabsi M, Echarrab M, Elouannani M, Errougani A, Amraoui M, Chkoff MR. Undifferentiated pancreatic carcinoma with osteoclast-like giant cells: a case report. J Gastrointest Cancer 2014;45 Suppl 1:96-8.

6. Sakhi R, Hamza A, Khurram MS, Ibrar W, Mazzara P. Undifferentiated carcinoma of the pancreas with osteoclastlike giant cells reported in an asymptomatic patient: a rare case and literature review. Autops Case Rep 2017;7:51-7.

7. Sah SK, Li Y, Li Y. Undifferentiated carcinoma of the pancreas with osteoclast-like giant cells: a rare case report and review of the literature. Int J Clin Exp Pathol 2015;8:11785-91.

8. Muraki T, Reid MD, Basturk O, Jang KT, Bedolla G, Bagci P, Mittal P, Memis B, Katabi N, Bandyopadhyay S, Sarmiento JM, Krasinskas A, Klimstra DS, Adsay V. Undifferentiated Carcinoma with Osteoclastic Giant Cells of the Pancreas: Clinicopathologic Analysis of 38 Cases Highlights a More Protracted Clinical Course Than Currently Appreciated. Am J Surg Pathol 2016;40:1203-16.

9. Singhal A, Shrago SS, Li SF, Huang Y, Kohli V. Giant cell tumor of the pancreas: a pathological diagnosis with poor prognosis. Hepatobiliary Pancreat Dis Int 2010;9:433-7.

10. Reid MD, Muraki T, HooKim K, Memis B, Graham RP, Allende D, Shi J, Schaeffer DF, Singh R, Basturk O, Adsay V. Cytologic features and clinical implications of undifferentiated carcinoma with osteoclastic giant cells of the pancreas: An analysis of 15 cases. Cancer Cytopathol 2017;125:563-75.

11. Lee ES, Lee JM. Imaging diagnosis of pancreatic cancer: a state-of-the-art review. World J Gastroenterol 2014;20:7864-77. 